\title{
THE WAVELET TRANSFORM OF DISTRIBUTIONS
}

\author{
RAM S. PATHAK
}

(Received January 27, 2003, revised October 15, 2003)

\begin{abstract}
The continuous wavelet transform is extended to certain distributions and continuity results are obtained. Boundedness results in a generalized Sobolev space, Besov space and Lizorkin-Triebel space are given.
\end{abstract}

1. Introduction. The wavelet transform of $\phi$ with respect to the wavelet $\psi$ is defined by

$$
\tilde{\phi}(b, a)=(W \phi)(b, a)=\int_{\boldsymbol{R}^{n}} \phi(t) \overline{\psi((t-b) / a)} d t / a^{n},
$$

provided the integral exists, where $b \in \boldsymbol{R}^{n}$ and $a>0$. Sometimes it is assumed that $a \in \boldsymbol{R} \backslash\{0\}$. If $\phi \in L^{2}\left(\boldsymbol{R}^{n}\right)$ and $\psi \in L^{2}\left(\boldsymbol{R}^{n}\right)$, then using the Parseval formula for Fourier transforms, (1.1) can be written in the following form (cf. [2, p. 9]):

$$
(W \phi)(b, a)=(2 \pi)^{-n} \int_{\boldsymbol{R}^{n}} e^{i(\omega, b)} \overline{\hat{\psi}(a \omega)} \hat{\phi}(\omega) d \omega .
$$

This form of the wavelet transform is very similar to that of a pseudo-differential operator with symbol $\sigma(a, \omega)=\overline{\hat{\psi}(a \omega)}$. Hence the theory of the wavelet transform (1.2) can be developed in a manner similar to that of the pseudo-differential operator (cf. [11]).

A reconstruction formula for (1.1) is given by

$$
\phi(x)=W^{-1}[\tilde{\phi}(b, a)](x)=\left(C_{\psi}\right)^{-1} \int_{\boldsymbol{R}_{+}} \int_{\boldsymbol{R}^{n}} \tilde{\phi}(b, a) \overline{\psi((x-b) / a)} d a d b / a^{n+1},
$$

where

$$
C_{\psi}=\int_{\boldsymbol{R}^{n}}|\hat{\psi}(\omega)|^{2}|\omega|^{-n} d \omega>0 .
$$

It has been proved by Perrier and Basdevant [6] that for $\psi$ satisfying the admissibility condition (1.4) with $n=1$, the wavelet transform is a linear bounded operator:

$$
W: L^{p}(\boldsymbol{R}) \rightarrow L^{p}\left(\boldsymbol{R}, L^{2}\left(\boldsymbol{R}_{+}, d a / a\right)\right):=W^{p}, \quad 1<p<\infty,
$$

and we have

$$
\|\tilde{f}(b, a)\|_{W^{p}}:=\left(\int_{\boldsymbol{R}}\left(\int_{\boldsymbol{R}_{+}}|\tilde{f}(b, a)|^{2} d a / a\right)^{p / 2} d b\right)^{1 / p} \leq A_{p}\|f\|_{L^{p}},
$$

2000 Mathematics Subject Classification. Primary 42C40; Secondary 46F12.

Key words and phrases. Continuous wavelet transform, distributions, Sobolev space, Besov space, LizorkinTriebel space.

The work is supported by CSIR Grant No. 25(0103)/99/EMR-II. 
where the constant $A_{p}$ depends on $p$ and $\psi$. Moreover, the inverse wavelet transform given by (1.3) with $n=1$ is a linear and bounded operator:

$$
W^{-1}: L^{p}\left(\boldsymbol{R}, L^{2}\left(\boldsymbol{R}_{+}, d a / a\right)\right) \rightarrow L^{p}(\boldsymbol{R})
$$

and we have for some constant $D>0$,

$$
\|f\|_{L^{p}} \leq D\|\tilde{f}(b, a)\|_{W^{p}}, \quad 1<p<\infty .
$$

We shall use the following Parseval relation (cf. [6]). For $f \in L^{p}(\boldsymbol{R}), g \in L^{p^{\prime}}(\boldsymbol{R})$ with $1<p<\infty, 1 / p+1 / p^{\prime}=1$ and real wavelet $\psi$ satisfying (1.4) we have

$$
\int_{\boldsymbol{R}} f(x) \overline{g(x)} d x=\left(C_{\psi}\right)^{-1} \int_{\boldsymbol{R}}\left(\int_{\boldsymbol{R}_{+}} \tilde{f}(b, a) \overline{\tilde{g}(b, a)} d a / a\right) d b .
$$

Now, we define the Schwartz space $S\left(\boldsymbol{R}^{n}\right)$. An infinitely differentiable complex valued function $\phi$ on $\boldsymbol{R}^{n}$ is said to belong to the test function space $S\left(\boldsymbol{R}^{n}\right)$ if

$$
\gamma_{m, \beta}(\phi)=\sup _{x \in \boldsymbol{R}^{n}}\left|(1+|x|)^{m} D_{x}^{\beta} \phi(x)\right|<\infty
$$

for all $m \in N_{0}$ and $\beta \in N_{0}^{n}$. Here $D_{x}^{\beta}$ denotes $\left(\partial / \partial x_{1}\right)^{\beta_{1}}\left(\partial / \partial x_{2}\right)^{\beta_{2}} \cdots\left(\partial / \partial x_{n}\right)^{\beta_{n}}$ with $\beta=$ $\left(\beta_{1}, \beta_{2}, \ldots, \beta_{n}\right)$. The dual of the space $S$ is the space $S^{\prime}$ of tempered distributions (cf. [5])

We also recall the definitions of the Besov space $B_{p, q}^{s}$ and the Lizorkin-Triebel space $F_{p, q}^{s}$ from [10, p. 45].

Let $\Phi\left(\boldsymbol{R}^{n}\right)$ be the collection of all systems $\left\{\phi_{j}\right\}_{j=0}^{\infty} \in S\left(\boldsymbol{R}^{n}\right)$ such that

$$
\left\{\begin{array}{l}
\operatorname{supp} \phi_{0} \subset\{x ;|x| \leq 2\} \\
\operatorname{supp} \phi_{j} \subset\left\{x ; 2^{j-1} \leq|x| \leq 2^{j+1}\right\}, \quad j=1,2,3, \ldots
\end{array}\right.
$$

that for every multiindex $\alpha$ there exists a positive number $C_{\alpha}$ with

$$
2^{j|\alpha|}\left|D^{\alpha} \phi_{j}(x)\right| \leq C_{\alpha} \quad \text { for all } j=0,1,2, \ldots \text { and all } x \in \boldsymbol{R}^{n}
$$

and that

$$
\sum_{j=0}^{\infty} \phi_{j}(x)=1 \quad \text { for every } x \in \boldsymbol{R}^{n} .
$$
$\Phi\left(\boldsymbol{R}^{n}\right)$.

Definition 1.1. Let $-\infty<s<\infty$ and $0<q \leq \infty$. Assume that $\left\{\phi_{j}(x)\right\}_{j=0}^{\infty} \in$

(i) If $0<p \leq \infty$, then

$$
B_{p, q}^{s}\left(\boldsymbol{R}^{n}\right)=\left\{f ; f \in S^{\prime}\left(\boldsymbol{R}^{n}\right),\left\|f\left|B_{p, q}^{s}\left(\boldsymbol{R}^{n}\right)\|=\| 2^{s j} F^{-1} \phi_{j} F f\right| \ell_{q}\left(L_{p}\left(\boldsymbol{R}^{n}\right)\right)\right\|<\infty\right\} .
$$

(ii) If $0<p<\infty$, then

$$
F_{p, q}^{s}\left(\boldsymbol{R}^{n}\right)=\left\{f ; f \in S^{\prime}\left(\boldsymbol{R}^{n}\right),\left\|f\left|F_{p, q}^{s}\left(\boldsymbol{R}^{n}\right)\|=\| 2^{s j} F^{-1} \phi_{j} F f\right| L_{p}\left(\boldsymbol{R}^{n}, \ell_{q}\right)\right\|<\infty\right\} .
$$

For further properties of these spaces we refer to [10]. 
Imposing suitable conditions on $\hat{\psi}$ we investigate the continuity of the wavelet transform (1.2) and define the generalized wavelet transform of certain tempered distributions in Section 2. Using inequalities (1.5) and (1.6) we investigate the wavelet transform of certain $\left(D_{L^{p}}\right)^{\prime}$ type distributions of Schwartz in Section 3. Using the definition (1.2) we study in Section 4 the wavelet transform on a certain $L^{p}$-Sobolev space. In Section 5 a multiplier theorem of Triebel is applied to (1.2) to obtain some boundedness results for the wavelet transform in the Besov space $B_{p, q}^{s}$ and the Triebel-Lizorkin space $F_{p, q}^{s}$ for all $s \in \boldsymbol{R}$ and $0<p, q \leq \infty$. The paper extends some of the results of Perrier and Basdevant [6] and Moritoh [4].

The author would like to express his sincere gratitude to the referee for his valuable advice.

2. The wavelet transform of tempered distributions. In this section we need the test function space $\tilde{S}\left(\boldsymbol{R}^{n} \times \boldsymbol{R}_{+}\right)$defined to be the space of all functions $\phi \in C^{\infty}\left(\boldsymbol{R}^{n} \times \boldsymbol{R}_{+}\right)$ such that for $\ell, k \in N_{0}$ and $\alpha, \beta \in N_{0}^{n}$,

$$
\gamma_{\ell, \alpha, k, \beta}(\phi)=\sup _{\substack{(b, a) \in \boldsymbol{R}^{n} \times \boldsymbol{R}_{+} \\ \ell+|\alpha| \leq k+|\beta|}}\left|a^{\ell} b^{\alpha}(\partial / \partial a)^{k} D_{b}^{\beta} \phi(b, a)\right|<\infty .
$$

Clearly, the Schwartz space $S\left(\boldsymbol{R}^{n} \times \boldsymbol{R}_{+}\right)$is contained in $\tilde{S}\left(\boldsymbol{R}^{n} \times \boldsymbol{R}_{+}\right)$.

THEOREM 2.1. Let $\psi \in S\left(\boldsymbol{R}^{n}\right)$. Then the wavelet transform $(W \phi)(b, a)$ is a continuous linear map of $S\left(\boldsymbol{R}^{n}\right)$ into $\tilde{S}\left(\boldsymbol{R}^{n} \times \boldsymbol{R}_{+}\right)$.

Proof. For $\ell, k \in N_{0}$ and $\alpha, \beta \in N_{0}^{n}$, we have after differentiation and integration by parts,

$$
\begin{aligned}
a^{\ell} b^{\alpha}(\partial / \partial a)^{k} D_{b}^{\beta}(W \phi)(b, a) \\
=(2 \pi)^{-n} b^{\alpha} D_{b}^{\beta} \int_{\boldsymbol{R}^{n}} e^{i(b, \omega)} \hat{\phi}(\omega)\left\{a^{\ell}(\partial / \partial a)^{k} \overline{\hat{\psi}(a \omega)}\right\} d \omega \\
=(2 \pi)^{-n} b^{\alpha} \int_{\boldsymbol{R}^{n}} e^{i(b, \omega)}(i \omega)^{\beta} \hat{\phi}(\omega) i^{\ell+k}\left(\omega^{-\ell} \int_{\boldsymbol{R}^{n}} e^{i(\omega, a y)}\left(D_{y}\right)^{\ell}\left[(\omega, y)^{k} \overline{\psi(y)}\right] d y\right) d \omega \\
=(2 \pi)^{-n} b^{\alpha} \int_{\boldsymbol{R}^{n}} e^{i(b, \omega)} i^{|\beta|+\ell+k} \omega^{\beta-\ell} \hat{\phi}(\omega)\left(\int_{\boldsymbol{R}^{n}} e^{i(\omega, a y)} D_{y}^{\ell}\left[\sum_{|\lambda|=k}(k ! / \lambda !) \omega^{\lambda} y^{\lambda} \bar{\psi}(y)\right] d y\right) d \omega \\
=(2 \pi)^{-n} i^{\ell+k+|\alpha+\beta|} \int_{\boldsymbol{R}^{n}} e^{i(b, \omega)} \sum_{|\lambda|=k}(k ! / \lambda !) \sum_{\delta \leq \alpha}\left(\begin{array}{l}
\alpha \\
\delta
\end{array}\right)\left(D_{\omega}\right)^{\alpha-\delta}\left(\omega^{\beta+\lambda-\ell} \hat{\phi}(\omega)\right) \\
\quad \times\left(\int_{\boldsymbol{R}^{n}}(i a y)^{\delta}\left(D_{y}\right)^{\ell}\left[y^{\lambda} \overline{\psi(y)}\right] e^{i(\omega, a y)} d y\right) d \omega \\
=(2 \pi)^{-n} i^{\ell+k+|\alpha+\beta+2 \delta|} \int_{\boldsymbol{R}^{n}} e^{i(b, \omega)} \sum_{|\lambda|=k}(k ! / \lambda !) \sum\left(\begin{array}{l}
\alpha \\
\delta
\end{array}\right) \omega^{-\delta} D_{\omega}^{\alpha-\delta}\left(\omega^{\beta+\lambda-\ell} \hat{\phi}(\omega)\right)
\end{aligned}
$$




$$
\begin{aligned}
& \times \int_{\boldsymbol{R}^{n}}\left(D_{y}\right)^{\delta} y^{\delta}\left(D_{y}\right)^{\ell}\left(y^{\lambda} \hat{\psi}(y)\right) e^{i(\omega, a y)} d y d \omega \\
= & (2 \pi)^{-n} i^{\ell+k+1+|\alpha+\beta+2 \delta|} \sum_{|\lambda|=k}(k ! / \lambda !) \sum_{\delta}\left(\begin{array}{c}
\alpha \\
\delta
\end{array}\right) \sum_{\rho}\left(\begin{array}{c}
\alpha-\delta \\
\rho
\end{array}\right) \\
& \times \int_{\boldsymbol{R}^{n}} e^{i(b, \omega)} A(\beta, \ell, \lambda, \rho) \omega^{\beta+\lambda-\ell-\rho-\delta} D_{\omega}^{\alpha-\delta-\rho} \hat{\phi}(\omega) \\
& \times\left(\sum_{\gamma \leq \delta}\left(\begin{array}{c}
\delta \\
\gamma
\end{array}\right) A^{\prime}(\delta, \gamma) \int_{\boldsymbol{R}^{n}} y^{\delta-\gamma} D_{y}^{\ell+\delta-\gamma}\left(y^{\lambda} \bar{\psi}(y)\right) e^{i(\omega, a y)} d y\right) d \omega .
\end{aligned}
$$

Therefore, for $|\beta|+k \geq|\alpha|+\ell$, we can write

$$
\begin{aligned}
\left|a^{\ell} b^{\alpha}(\partial / \partial a)^{k} D_{b}^{\beta} W \phi(b, a)\right| & \\
\leq & (2 \pi)^{-n} \sum_{|\lambda|=k}(k ! / \lambda !) \sum_{\delta \leq \alpha}\left(\begin{array}{l}
\alpha \\
\delta
\end{array}\right) \sum_{\rho \leq \alpha-\delta}\left(\begin{array}{c}
\alpha-\delta \\
\rho
\end{array}\right) \sum_{\gamma \leq \delta}\left(\begin{array}{l}
\delta \\
\gamma
\end{array}\right) \sum_{\tau \leq \ell+\delta-\gamma}\left(\begin{array}{c}
\ell+\delta-\gamma \\
\tau
\end{array}\right) \\
& \times|A(\beta, \ell, \lambda, \rho)|\left|A^{\prime}(\delta, \gamma)\right|\left|A^{\prime \prime}(\lambda, \tau, \psi)\right| \\
& \times \int_{\boldsymbol{R}^{n}}(1+|\omega|)^{|\beta|+k-\ell-|\rho|-|\delta|+n+1}\left|D_{\omega}^{\alpha-\delta-\rho} \hat{\phi}(\omega)\right| d \omega /(1+|\omega|)^{n+1} \\
& \times \int_{\boldsymbol{R}^{n}}(1+|y|)^{k+|\delta|-|\tau|-|\gamma|+n+1}\left|D_{y}^{\ell+\delta-\gamma+\tau} \bar{\psi}(y)\right| d y /(1+|y|)^{n+1},
\end{aligned}
$$

so that

$$
\begin{aligned}
\gamma_{\ell, \gamma, k, \beta}(W \phi) \leq & \sum_{|\lambda|=k}(k ! / \lambda !) \sum_{\delta \leq \alpha}\left(\begin{array}{l}
\alpha \\
\delta
\end{array}\right) \sum_{\rho \leq \alpha-\delta}\left(\begin{array}{c}
\alpha-\delta \\
\rho
\end{array}\right) \sum_{\gamma \leq \delta}\left(\begin{array}{l}
\delta \\
\gamma
\end{array}\right) \sum_{\tau \leq \ell+\delta-\gamma}\left(\begin{array}{c}
\ell+\delta-\gamma \\
\tau
\end{array}\right) \\
& \times B(\alpha, \beta, \gamma, \tau, \lambda, \delta, \rho, \ell, n) \gamma|\beta|+k-\ell-|\rho|-|\delta|+n+1, \alpha-\delta-\rho(\hat{\phi}) \\
& \times \gamma_{k+|\delta|-|\gamma|-|\tau|+n+1, \ell+\delta+\tau-\gamma}(\psi) .
\end{aligned}
$$

Thus $W \phi(b, a) \in \tilde{S}\left(\boldsymbol{R}^{n} \times \boldsymbol{R}_{+}\right)$, and from the above inequality the continuity of $W \phi$ also follows.

In view of the above theorem the generalized wavelet transform $W^{\prime} T$ of $T \in \tilde{S}^{\prime}$, the dual of $\tilde{S}\left(\boldsymbol{R}^{n} \times \boldsymbol{R}_{+}\right)$, can be defined by

$$
\left\langle W^{\prime} T, \phi\right\rangle=\langle T, W \phi\rangle, \quad \phi \in S\left(\boldsymbol{R}^{n}\right) .
$$

Using duality arguments we have:

THEOREM 2.2. The generalized wavelet transform $W^{\prime}: \tilde{S}^{\prime} \rightarrow S^{\prime}$ is linear and continuous.

We can also analyse the wavelet transform by imposing a condition on the Fourier transform of the wavelet $\psi$ as follows:

Assume that $\hat{\psi} \in C^{\infty}\left(\boldsymbol{R}^{n}\right)$ such that

$$
\|\hat{\psi}\|_{\alpha}^{m, \rho}=\sup _{\xi}(1+|\xi|)^{-m+\rho|\alpha|}\left|D^{\alpha} \hat{\psi}(\xi)\right|<\infty
$$


where $m \in \boldsymbol{R}, 0 \leq \rho \leq 1, \alpha \in \boldsymbol{N}_{0}^{n}$. Let us define the function space $S_{1}\left(\boldsymbol{R}^{n} \times \boldsymbol{R}_{+}\right)$to be the set of all $C^{\infty}$-functions $\phi$ on $\boldsymbol{R}^{n} \times \boldsymbol{R}_{+}$such that for $\ell, k \in \boldsymbol{N}_{0}$ and $\alpha, \beta \in \boldsymbol{N}_{0}^{n}$,

$$
\gamma_{\ell, \alpha, k, \beta}(\phi)=\sup _{\substack{(b, a) \in \boldsymbol{R}^{n} \times \boldsymbol{R}_{+} \\ \ell+|\alpha \leq k+| \beta| \\\ell+| \alpha \mid \leq m}}\left|a^{\ell} b^{\alpha}(\partial / \partial a)^{k} D_{b}^{\beta} \phi(b, a)\right|<\infty .
$$

THEOREM 2.3. Assume that the wavelet $\psi$ satisfies (2.3). Then the wavelet transform $W$ is a continuous linear map of $S\left(\boldsymbol{R}^{n}\right)$ into $S_{1}\left(\boldsymbol{R}^{n} \times \boldsymbol{R}_{+}\right)$, and the generalized wavelet transform is a continuous linear map of $S_{1}^{\prime}\left(\boldsymbol{R}^{n} \times \boldsymbol{R}_{+}\right)$into $S^{\prime}\left(\boldsymbol{R}^{n}\right)$.

PROOF. Proceeding as in the proof of Theorem 2.1 we can write

$$
\begin{aligned}
& \left|a^{\ell} b^{\alpha}(\partial / \partial a)^{k} D_{b}^{\beta}(W \phi)(b, a)\right| \\
& \leq(2 \pi)^{-n} a^{\ell} \int_{\boldsymbol{R}^{n}} \sum_{|\lambda|=k}(k ! / \lambda !) \sum_{\gamma \leq \alpha}\left(\begin{array}{l}
\alpha \\
\gamma
\end{array}\right)\left|\left[D_{u}^{\gamma} D_{u}^{\lambda} \overline{\hat{\psi}(u)}\right]_{u=a \omega}\right| a^{|\gamma|}|\omega|^{|\lambda|} \\
& \times \sum_{\delta \leq \alpha-\gamma}\left(\begin{array}{c}
\alpha-\gamma \\
\delta
\end{array}\right) A(\beta, \delta)|\omega|^{|\beta-\delta|}\left|D_{\omega}^{\alpha-\gamma-\delta} \hat{\phi}(\omega)\right| d \omega \\
& \leq(2 \pi)^{-n} \sum_{|\lambda|=k}(k ! / \lambda !) \sum_{\gamma \leq \alpha} \sum_{\delta \leq \alpha-\gamma}\left(\begin{array}{l}
\alpha \\
\gamma
\end{array}\right)\left(\begin{array}{c}
\alpha-\delta \\
\delta
\end{array}\right) A(\beta, \delta) a^{|\gamma|+\ell} \\
& \times \int_{\boldsymbol{R}^{n}}|\omega|^{|\lambda|+|\beta-\delta|}(1+a|\omega|)^{m-\rho(|\gamma|+|\lambda|)}\left|D_{\omega}^{\alpha-\gamma-\delta} \hat{\phi}(\omega)\right| d \omega \\
& \leq(2 \pi)^{-n} \sum_{|\lambda|=k} \sum_{\gamma \leq \alpha} \sum_{\delta \leq \alpha-\gamma}(k ! / \lambda !)\left(\begin{array}{l}
\alpha \\
\gamma
\end{array}\right)\left(\begin{array}{c}
\alpha-\delta \\
\delta
\end{array}\right) A(\beta, \delta) \\
& \times \int_{\boldsymbol{R}^{n}}(1+a|\omega|)^{|\gamma|+\ell+m-\rho(|\gamma|+k)}|\omega|^{k+|\beta|-|\delta|-|\gamma|-\ell}\left|D_{\omega}^{\alpha-\gamma-\delta} \hat{\phi}(\omega)\right| d \omega \\
& \leq(2 \pi)^{-n} \sum_{|\lambda|=k} \sum_{\gamma \leq \alpha} \sum_{\delta \leq \alpha-\gamma}(k ! / \lambda !)\left(\begin{array}{l}
\alpha \\
\gamma
\end{array}\right)\left(\begin{array}{c}
\alpha-\delta \\
\delta
\end{array}\right) A(\beta, \delta) \\
& \times \int_{\boldsymbol{R}^{n}}(1+a|\omega|)^{k+|\beta|-|\delta|-|\gamma|-\ell+n+1}\left|D_{\omega}^{\alpha-\gamma-\delta} \hat{\phi}(\omega)\right| d \omega(1+|\omega|)^{-n-1}
\end{aligned}
$$

for $|\gamma|+\ell+m-\rho(|\gamma|+k)<0$. Therefore, for $m<-\ell-|\alpha|$ and $\ell+|\alpha| \leq k+|\beta|$, we have

$$
\begin{aligned}
\left|a^{\ell} b^{\alpha}(\partial / \partial a)^{k} D_{b}^{\beta}(W \phi)(b, a)\right| \leq & (2 \pi)^{-n} \sum_{|\lambda|=k} \sum_{\gamma \leq \alpha} \sum_{\delta \leq \alpha-\gamma}(k ! / \lambda !)\left(\begin{array}{l}
\alpha \\
\gamma
\end{array}\right)\left(\begin{array}{c}
\alpha-\gamma \\
\delta
\end{array}\right) \\
& \times A(\beta, \delta, n) \gamma_{k+|\beta|+|\delta|-|\gamma|-\ell+n+1, \alpha-\gamma-\delta}(\hat{\phi}) .
\end{aligned}
$$

From this we conclude that the wavelet transform is a continuous linear map of $S\left(\boldsymbol{R}^{n}\right)$ into $S_{1}\left(\boldsymbol{R}^{n} \times \boldsymbol{R}_{+}\right)$.

As in Theorem 2.1 we define the generalized wavelet transform $W^{\prime}$ of $T \in\left(S_{1}\right)^{\prime}$, the dual of $S_{1}\left(\boldsymbol{R}^{n} \times \boldsymbol{R}_{+}\right)$, by (2.2) and get the second part of the theorem. 
3. The wavelet transform of $\left(D_{L^{p}}\right)^{\prime}$-type distributions. In this section, to simplify the analysis, we consider the wavelet transform (1.1) for $b \in \boldsymbol{R}$ and $a>0$. We define a suitable test function space.

An infinitely differentiable complex-valued function $\phi$ on $\boldsymbol{R}$ is said to belong to the test function space $F_{p}(\boldsymbol{R})$ if

$$
\gamma_{k}^{p}(\phi)=\left(\int_{\boldsymbol{R}}\left|(x \partial / \partial x)^{k} \phi(x)\right|^{p} d x\right)^{1 / p}<\infty, \quad 1 \leq p<\infty
$$

for every $k \in N_{0}$. A variant of $F_{p}(0, \infty)$, denoted by $F_{p, 0}(0, \infty)$ was studied by McBride [3].

Another space with which we shall be concerned is $G_{p}\left(\boldsymbol{R} \times \boldsymbol{R}_{+}\right)$, defined for $b \in \boldsymbol{R}, a \in$ $\boldsymbol{R}_{+}$and $1 \leq p<\infty$, by

$$
\begin{aligned}
G_{p}\left(\boldsymbol{R} \times \boldsymbol{R}_{+}\right)=\{\phi & \in C^{\infty}\left(\boldsymbol{R} \times \boldsymbol{R}_{+}\right) ; \beta_{k}^{p}(\phi)= \\
& \left(\int_{\boldsymbol{R}} \int_{\boldsymbol{R}_{+}}\left(\left|(a \partial / \partial a+b \partial / \partial b+1)^{k} \phi(b, a)\right|^{2} \frac{d a}{a}\right)^{p / 2} d b\right)^{1 / p}<\infty \\
& \text { for all } \left.k \in N_{0}\right\} .
\end{aligned}
$$

Here we note that a differentiable function $\psi$ satisfies the partial differential equation

$$
(a \partial / \partial a+b \partial / \partial b+1)[\psi((x-b) / a) / a]=-x \partial / \partial x[\psi((x-b) / a) / a],
$$

and therefore, in general, we have

$$
\begin{aligned}
& (a \partial / \partial a+b \partial b+1)^{k}[\psi((x-b) / a) / a] \\
& \quad=(-1)^{k}(x \partial / \partial x)^{k}[\psi((x-b) / a) / a], \quad k \in N_{0} .
\end{aligned}
$$

Now, we prove the following:

THEOREM 3.1. Let $\phi \in F_{p}(\boldsymbol{R})$ and $\psi \in S(\boldsymbol{R})$ with (1.4). Then, for $1<p<\infty$, the wavelet transform $W \phi$ defined by (1.1) is a one-to-one linear continuous map from $F_{p}(\boldsymbol{R})$ onto $G_{p}\left(\boldsymbol{R} \times \boldsymbol{R}_{+}\right)$and $W^{-1}$ is given by (1.3).

Proof. Let us assume that the real wavelet $\psi \in S(\boldsymbol{R}) \subset L^{1}(\boldsymbol{R}) \cap L^{2}(\boldsymbol{R})$ and $\phi \in$ $F_{p}(\boldsymbol{R}) \subset L^{p}(\boldsymbol{R})$. Then differentiating under the integral sign in (1.1) with $n=1$, and using the relation (3.3) and integrating by parts we can show that

$$
\begin{aligned}
(a \partial / \partial a+b \partial / \partial b+1) W(b, a) & =\int_{\boldsymbol{R}}(a \partial / \partial a+b \partial / \partial b+1) \overline{\psi((x-b) / a)} a^{-1} \phi(x) d x \\
& =-\int_{\boldsymbol{R}}(x \partial / \partial x) \overline{\psi((x-b) / a)} a^{-1} \phi(x) d x \\
& =\int_{\boldsymbol{R}}(x \partial / \partial x+1) \phi(x) \overline{\psi((x-b) / a)} a^{-1} d x .
\end{aligned}
$$


Therefore, in general, we have

$$
\begin{aligned}
(a \partial / \partial a+b \partial / \partial b+1)^{k} W(b, a) & =(a \partial / \partial a+b \partial / \partial b+1)^{k}(W \phi)(b, a) \\
& =W\left[(x \partial / \partial x+1)^{k} \phi\right](b, a) .
\end{aligned}
$$

Now, we apply the inequality (1.5) and get

$$
\begin{aligned}
\beta_{k}^{p}(W \phi) & =\left(\int_{\boldsymbol{R}}\left(\int_{\boldsymbol{R}_{+}}\left|W\left[(x \partial / \partial x+1)^{k} \phi\right](b, a)\right|^{2} \frac{d a}{a}\right)^{p / 2} d b\right)^{1 / p} \\
& \leq A_{p}\left\|(x \partial / \partial x+1)^{k} \phi\right\|_{L^{p}} \leq A_{p} \sum_{r=0}^{n}\left(\begin{array}{l}
n \\
r
\end{array}\right)\left\|(x \partial / \partial x)^{r} \phi\right\|_{L^{p}},
\end{aligned}
$$

where the constant $A_{p}$ depends on $p$ and $\psi$. From (3.6) we conclude that $W$ is a continuous linear mapping from $F_{p}(\boldsymbol{R})$ into $G_{p}\left(\boldsymbol{R} \times \boldsymbol{R}_{+}\right)$.

Next, differentiating (1.3) with respect to $x$ within the integral sign, using formula (3.3) and integrating by parts we obtain

$$
(x \partial / \partial x)^{k} \phi(x)=\left(C_{\psi}\right)^{-1} \int_{\boldsymbol{R}} \int_{\boldsymbol{R}_{+}}(a \partial / \partial a+b \partial / \partial b+1)^{k} W(b, a) \psi((x-b) / a) a^{-2} d a d b .
$$

Therefore, in view of the inequality (1.6) we obtain the following estimate, for $1<p<\infty$,

$$
\begin{aligned}
& \left\|(x d / d x)^{k} \phi(x)\right\|_{L^{p}} \\
& \quad \leq\left(D / C_{\psi}\right)\left(\int_{\boldsymbol{R}}\left(\int_{\boldsymbol{R}_{+}}\left|(a \partial / \partial a+b \partial / \partial b+1)^{k} W(b, a)\right|^{2} a^{-1} d a\right)^{p / 2} d b\right)^{1 / p} .
\end{aligned}
$$

From (3.7) we conclude that $W^{-1}$ is a continuous linear mapping of $G_{p}\left(\boldsymbol{R} \times \boldsymbol{R}_{+}\right)$into $F_{p}(\boldsymbol{R})$.

To prove that $W$ is one-to-one, assume that $W \phi=0$ for $\phi \in F_{p} \subset L^{p}$. Operating on both sides of this equation by $W^{-1}$ we get $W^{-1} W \phi=0$. Thus $\phi=0$. So $W \phi=0$ implies that $\phi=0$.

Now, we show that $W$ is onto. Let $\phi \in F_{p} \subset L_{p}$. Then $W \phi \in G_{p}\left(\boldsymbol{R} \times \boldsymbol{R}_{+}\right)$, but $W^{-1}$ $(W \phi)=\phi$. Therefore, for every $\phi \in F_{p}$ there exists $W \phi \in G_{p}\left(\boldsymbol{R} \times \boldsymbol{R}_{+}\right)$that is mapped by $W^{-1}$ to $\phi$. Consequently, $W$ is onto also.

Clearly, $W$ is a one-to-one and onto mapping from $F_{p}(\boldsymbol{R})$ onto $G_{p}\left(\boldsymbol{R} \times \boldsymbol{R}_{+}\right)$. Therefore, $W^{-1}$ is defined on $G_{p}\left(\boldsymbol{R} \times \boldsymbol{R}_{+}\right)$. Since $W^{-1} W \phi=\phi$ for all $\phi \in F_{p}$ it follows that $W^{-1}$ is given by (1.3).

In analogy to the Parseval relation (1.7) for the classical wavelet transform $W$, we define the generalized wavelet transform $W^{\prime} f$ of $f \in F_{p}^{\prime}$ by the relation

$$
\langle f(x), \overline{\phi(x)}\rangle=\frac{1}{C_{\psi}}\left\langle\left(W^{\prime} f\right)(b, a), \overline{(W \phi)(b, a)}\right\rangle,
$$

where $\phi \in F_{p}(\boldsymbol{R})$ and $C_{\psi}$ is given by (1.4) with $n=1$.

Clearly $W^{\prime}$ is a linear functional on $G_{p}\left(\boldsymbol{R} \times \boldsymbol{R}_{+}\right)$; its continuity follows from Theorem 3.1. Thus $W^{\prime} f \in G_{p}^{\prime}$. 
Applying duality arguments to Theorem 3.1 we can also define the transform $W^{\wedge} g$ of $g \in G_{p}^{\prime}\left(\boldsymbol{R} \times \boldsymbol{R}_{+}\right)$by

$$
\left\langle W^{\wedge} g, \phi\right\rangle=\langle f, W \phi\rangle
$$

where $\phi \in F_{p}$. Then $W^{\wedge} g$ is a continuous linear map on $F_{p}$. If we set $g=W^{\prime} f$, then $W^{\wedge} g=f=\left(W^{\prime}\right)^{-1} g$, the inverse of the generalized wavelet transform of $g \in G_{p}^{\prime}$.

Thus we have the following:

THEOREM 3.2. Let $f \in F_{p}^{\prime}$ and $\psi \in S(\boldsymbol{R})$ satisfy (1.4) with $n=1$. Then, for $1<$ $p<\infty$, the generalized wavelet transform $W^{\prime} f$ defined by (3.8) is a one-to-one continuous linear map of $F_{p}^{\prime}$ onto $G_{p}^{\prime}$.

4. The wavelet transform on $L^{p}$-Sobolev space. One can measure the regularity of a function $\phi$ in the scale of an $L^{p}$-Sobolev space. For $-\infty<s<\infty$ and $1 \leq p<\infty$, then $L^{p}$-Sobolev sapce $H_{S}^{p}$ is defined to be set of all $\phi \in S^{\prime}\left(\boldsymbol{R}^{n}\right)$ such that

$$
\|\phi\|_{H_{s}^{p}}=\left\||\omega|^{s} \hat{\phi}\right\|_{L^{p}}
$$

where $\hat{\phi}=F \phi$.

We are also concerned with the space $W_{\rho}^{p^{\prime}, s}$ of all measurable functions $\phi$ on $\boldsymbol{R}^{n} \times \boldsymbol{R}_{+}$ such that

$$
\begin{aligned}
\|\phi(\cdot, \cdot)\|_{W_{p}^{p^{\prime}, s}}= & \left(\int_{0}^{\infty}\left(\int_{\boldsymbol{R}^{n}}|\phi(b, a)|^{p} d b\right)^{p^{\prime} / p} a^{-s-1} d a\right)^{1 / p^{\prime}}<\infty, \\
& 1 \leq p, p^{\prime}<\infty, \quad s \in \boldsymbol{R} .
\end{aligned}
$$

Now, from (1.2) it follows that

$$
F[W(\cdot, a)](\omega)=\overline{\hat{\psi}(a \omega)} \hat{\phi}(\omega) .
$$

Using the Haussdorff-Young inequality [8, p. 178] for $1 \leq p \leq 2,1 / p+1 / p^{\prime}=1$, we have

$$
\begin{aligned}
\left(\int_{\boldsymbol{R}^{n}}|\overline{\hat{\psi}(a \omega)} \hat{\phi}(\omega)|^{p^{\prime}} d \omega\right)^{1 / p^{\prime}} & =\left(\int_{\boldsymbol{R}^{n}}|F W(\cdot, a)|^{p^{\prime}} d \omega\right)^{1 / p^{\prime}} \\
& \leq C_{p, n}\left(\int_{\boldsymbol{R}^{n}}|W(b, a)|^{p} d b\right)^{1 / p}
\end{aligned}
$$

where $C_{p, n}>0$ is a constant. Multiplying by $a^{-s-1} d a$ and integrating from 0 to $\infty$ we get

$$
\left.\int_{0}^{\infty} a^{-s-1} d a \int_{\boldsymbol{R}^{n}} \overline{\mid \hat{\psi}(a \omega)} \hat{\phi}(\omega)\right|^{p^{\prime}} d \omega \leq\left(C_{p, n}\right)^{p^{\prime}} \int_{0}^{\infty}\left(|W(b, a)|^{p} d b\right)^{p^{\prime} / p} a^{-s-1} d a
$$

so that

$$
C_{\psi}^{s, p^{\prime}} \int_{\boldsymbol{R}^{n}}|\omega|^{s}|\hat{\phi}(\omega)|^{p} d \omega \leq\left(C_{p, n}\right)^{p^{\prime}}\|\tilde{\phi}(b, a)\|_{W_{p}^{p^{\prime}, s}}^{p},
$$


where

$$
C_{\psi}^{s, p^{\prime}}=\int_{\boldsymbol{R}^{n}}|\hat{\psi}(a \omega)|^{p^{\prime}}(a|\omega|)^{-s} d a / a>0
$$

is assumed to be independent of $\omega$. Thus

$$
\|\phi\|_{H_{s}^{p^{\prime}}} \leq\left(C_{p, n} /\left(C_{\psi}^{s, p^{\prime}}\right)^{1 / p^{\prime}}\right)\|\tilde{\phi}(b, a)\|_{W_{p}^{p^{\prime}, s}} .
$$

Furthermore, using the Haussdorff-Young inequality again, from (4.3) we also have

$$
\begin{aligned}
& \left.\left(\int_{\boldsymbol{R}^{n}}|W(b, a)|^{p^{\prime}} d b\right)^{1 / p^{\prime}}=\left.\left(\int_{\boldsymbol{R}^{n}} \mid F^{-1} \overline{[\hat{\psi}(a \omega)} \hat{\phi}(\omega)\right](b)\right|^{p^{\prime}} d b\right)^{1 / p^{\prime}} \\
& \leq D_{p^{\prime}, n}\left(\int_{\boldsymbol{R}^{n}}|\bar{\psi}(a \omega) \hat{\phi}(\omega)|^{p} d \omega\right)^{1 / p}, \quad D_{p^{\prime}, n}>0 .
\end{aligned}
$$

Therefore,

$$
\begin{aligned}
\int_{0}^{\infty} a^{-s-1}\left(\int_{\boldsymbol{R}^{n}}|W(b, a)|^{p^{\prime}} d b\right)^{p / p^{\prime}} d a & \leq\left(D_{p^{\prime}, n}\right)^{p} \int_{0}^{\infty} a^{-s-1}\left(\int_{\boldsymbol{R}^{n}}|\hat{\psi}(a \omega) \hat{\phi}(\omega)|^{p} d \omega\right) d a \\
& =\left(D_{p^{\prime}, n}\right)^{p} \int_{\boldsymbol{R}^{n}}|\hat{\phi}(\omega)|^{p}|\omega|^{s} d \omega C_{\psi}^{s, p}
\end{aligned}
$$

where $C_{\psi}^{s, p}$ is given by (4.6). Thus

$$
\|\tilde{\phi}(b, a)\|_{W_{p^{\prime}}^{p, s}} \leq D_{p^{\prime}, n}\left(C_{\psi}^{s, p}\right)^{1 / p}\|\phi\|_{H_{s}^{p}}, \quad 1 / p+1 / p^{\prime}=1 .
$$

From (4.7) and (4.8) we get the following characterization of the $L^{p}$-Sobolev space in terms of the continuous wavelet transform, generalizing a result of Tchamitchian [9, p. 103] and Theorem 3.1 of Perrier and Basdewant [6].

THEOREM 4.1. Assume that the analysing wavelet satisfies the admissibility condition (4.6). Then the continuous wavelet transform is a bounded linear operator from $H_{s}^{p}\left(\boldsymbol{R}^{n}\right)$ into $W^{p^{\prime}, s}\left(\boldsymbol{R}^{n} \times \boldsymbol{R}_{+}\right)$for $1 \leq p \leq 2,1 / p+1 / p^{\prime}=1$ and for all $s \in \boldsymbol{R}$.

Moreover, for all $f \in H_{s}^{p}\left(\boldsymbol{R}^{n}\right)$, we have

$$
\|\phi\|_{H_{s}^{p^{\prime}}} \cong\|\tilde{\phi}(b, a)\|_{W_{p}^{p^{\prime}, s}},
$$

for all $s \in \boldsymbol{R}$ and $1 \leq p, p^{\prime} \leq 2,1 / p+1 / p^{\prime}=1$.

\section{Boundedness of the wavelet transform in Triebel-Lizorkin and Besov spaces.} Perrier and Basdevant [6] have obtained certain boundedness result for the continuous wavelet transform in Besov spaces. However, they restricted to the case $p, q \geq 1$ as they used Minkowski's inequality in their analysis. In this section using the following theorem on multipliers by Triebel [10] we obtain a boundedness result for the wavelet transform in the Besov space $B_{p, q}^{s}\left(\boldsymbol{R}^{n}\right)$ and the Triebel-Lizorkin sapce $F_{p, q}^{s}\left(\boldsymbol{R}^{n}\right)$ for all $s \in \boldsymbol{R}, 0<p, q \leq \infty$. 
THEOREM 5.1 (Triebel). Assume that $m: C^{\infty}\left(\boldsymbol{R}^{n}\right) \rightarrow C$ such that

$$
\|m\|_{N}=\sup _{|\alpha| \leq N} \sup _{x \in \boldsymbol{R}^{n}}\left(1+|x|^{2}\right)^{|\alpha| / 2}\left|D^{\alpha} m(x)\right|<\infty, \quad N \in \boldsymbol{N}_{0} .
$$

Let $-\infty<s<\infty$ and $0<q \leq \infty$. Let $A\left(\boldsymbol{R}^{n}\right)$ be either $B_{p, q}^{s}\left(\boldsymbol{R}^{n}\right)$ or $F_{p, q}^{s}\left(\boldsymbol{R}^{n}\right)$ with $0<p<\infty$. Then there exists a positive number $C$ such that

$$
\left\|F^{-1} m F f\left|A\|\leq C\| m\left\|_{N}\right\| f\right| A\right\|
$$

holds for all $m \in C^{\infty}\left(\boldsymbol{R}^{n}\right)$ and all $f \in A$ with

$$
N>|s|+3(n / p)+n+2 \text { for } B_{p, q}^{s}
$$

and

$$
N>|s|+3 n /(\min (p, q))+n+2 \text { for } F_{p, q}^{s} .
$$

The boundedness result for the wavelet transform in $B_{p, q}^{s}\left(\boldsymbol{R}^{n}\right)$ and $F_{p, q}^{s}\left(\boldsymbol{R}^{n}\right)$ is given by the following.

THEOREM 5.2. Let $-\infty<s<\infty$ and $0<q \leq \infty$. Let $f \in A$, where A denotes $B_{p, q}^{s}\left(\boldsymbol{R}^{n}\right)$ with $0<p \leq \infty$ or $F_{p, q}^{s}\left(\boldsymbol{R}^{n}\right)$ with $0<p<\infty$. Let $N$ denote the natural number such that

$$
N>|s|+3 n / p+n+2 \text { for } B_{p, q}^{s}
$$

and

$$
N>|s|+3 n /(\min (p, q))+n+2 \text { for } F_{p, q}^{s} .
$$

Assume that $\hat{\psi} \in C^{\infty}\left(\boldsymbol{R}^{n}\right)$ and (2.3) holds. Then there exists a positive number $C$ such that

$$
\left\|W_{\psi} f\left|A\left\|\leq C\left(1+a^{2}\right)^{N / 2} \sup _{|\alpha| \leq N}\right\| \hat{\psi}\left\|_{\alpha}^{\rho}\right\| f\right| A\right\|,
$$

for $m+(1-\rho) N \leq 0$.

ProOF. Assume that

$$
m(\xi)=\overline{\hat{\psi}(a \xi)}
$$

for $\xi \in \boldsymbol{R}^{n}, a>0$. Then

$$
\begin{aligned}
\|m\|_{N} & =\sup _{|\alpha| \leq N} \sup _{\xi \in \boldsymbol{R}^{n}}\left(1+|\xi|^{2}\right)^{|\alpha| / 2} \mid D^{\alpha} \overline{\hat{\psi}(a \xi) \mid} \\
& =\sup _{|\alpha| \leq N} \sup _{u}\left(a^{2}+|u|^{2}\right)^{|\alpha| / 2} \mid D_{u}^{\alpha} \overline{\hat{\psi}(u) \mid} \\
& \leq\left(1+a^{2}\right)^{N / 2} \sup _{|\alpha| \leq N} \sup _{u}\left(1+|u|^{2}\right)^{|\alpha| / 2}\left|D_{u}^{\alpha} \hat{\psi}(u)\right| \\
& \leq\left(1+a^{2}\right)^{N / 2} \sup _{|\alpha| \leq N} \sup _{u} \frac{(1+|u|)^{|\alpha|}}{(1+|u|)^{-m+\rho|\alpha|}}\left[(1+|u|)^{-m+\rho|\alpha|}\left|D^{\alpha} \hat{\psi}(u)\right|\right] \\
& \leq\left(1+a^{2}\right)^{N / 2} \sup _{|\alpha| \leq N}\|\hat{\psi}\|_{\alpha}^{m, \rho} .
\end{aligned}
$$


In view of Theorem 5.1 we have

$$
\left\|W_{\psi} f\left|A\|=\| F^{-1} \overline{\hat{\psi}(a .)} F f\right| A\right\|=\left\|F^{-1} m F f\left|A\|\leq C\| m\left\|_{N}\right\| f\right| A\right\| .
$$

Now, invoking the inequality (5.4) we get the desired estimate.

\section{REFERENCES}

[1] C. K. ChUI, Introduction to Wavelets, Academic Press, New York, 1992.

[2] M. Holschneider, Wavelets, An Analysis Tool, Clarendon Press, Oxford, 1995.

[ 3 ] A. C. MCBRIDE, Fractional Calculus and Integral Transforms of Generalized Functions, Pitman, London, 1979.

[ 4 ] S. MоRITOH, Wavelet transforms in Euclidian spaces-their relation with wavefront sets and Besov, TriebelZizorkin spaces, Tohoku Math. J. 47 (1995), 555-565.

[ 5 ] R. S. PAтHAK, Integral Transforms of Generalized Functions and their Applications, Gordon and Breach Science Publishers, Amsterdam, 1997.

[ 6 ] V. Perrier And C. Basdevant, Besov norms in terms of the continuous wavelet transform, Applications to structure functions, Math. Models Methods Appl. Sci. 6 (1996), 649-664.

[ 7 ] E. M. Stein, Harmonic Analysis: Real-Variable Methods, Orthogonality, and Oscillatory Integrals, Princeton University Press, Princeton, N.J., 1993.

[ 8 ] E. M. Stein and G. Weiss, Fourier Analysis on Euclidian Spaces, Princeton University Press, Princeton, N.J., 1975.

[ 9 ] P. TChamitchian, Wavelets, Functions, and Operators in Wavelets: Theory and Applications (ed. G. Erlebacher, M. Y. Hussainni and E. M. Jameson), 103-105, Oxford University, 1996.

[10] H. TRIEBEL, Theory of Function Spaces, Birkhauser-Verlag, Basel, 1983.

[11] M. W. Wong, An Introduction to Pseudo-Differential Operators, World Scientific, Singapore, 1991.

DEPARTMENT OF MATHEMATiCS

BANARAS HINDU UNIVERSITY

VARANASI 221005

INDIA

E-mail address: rspathak@banaras.ernet.in 